\title{
New model for the neutrino mass matrix
}

\author{
L. Lavoura \\ Universidade Técnica de Lisboa \\ Centro de Física das Interacções Fundamentais \\ Instituto Superior Técnico, 1049-001 Lisboa, Portugal
}

31 May 2000

\begin{abstract}
I suggest a model based on a softly broken symmetry $L_{e}-L_{\mu}-L_{\tau}$ and on Babu's mechanism for two-loops radiative generation of the neutrino masses. The model predicts that one of the physical neutrinos $\left(\nu_{3}\right)$ is massless and that its component along the $\nu_{e}$ direction $\left(U_{e 3}\right)$ is zero. Moreover, if the soft-breaking term is assumed to be very small, then the vacuum oscillations of $\nu_{e}$ have almost maximal amplitude and solve the solar-neutrino problem. New scalars are predicted in the $10 \mathrm{TeV}$ energy range, and a breakdown of $e-\mu-\tau$ universality should not be far from existing experimental bounds.
\end{abstract}

In a model without right-handed (singlet) neutrinos, the three weak-interaction-eigenstate neutrinos $\nu_{e}, \nu_{\mu}$, and $\nu_{\tau}$ may acquire $|\Delta I|=1$ Majorana masses given by the following term in the Lagrangian:

$$
\mathcal{L}_{\text {mass }}^{(\nu)}=\frac{1}{2}\left(\begin{array}{ccc}
\nu_{e}^{T} & \nu_{\mu}^{T} & \nu_{\tau}^{T}
\end{array}\right) C^{-1} \mathcal{M}\left(\begin{array}{c}
\nu_{e} \\
\nu_{\mu} \\
\nu_{\tau}
\end{array}\right)-\frac{1}{2}\left(\begin{array}{ccc}
\overline{\nu_{e}} & \overline{\nu_{\mu}} & \overline{\nu_{\tau}}
\end{array}\right) C \mathcal{M}^{*}\left(\begin{array}{c}
{\overline{\nu_{e}}}^{T} \\
\overline{\nu_{\mu}} \\
\overline{\nu_{\tau}} T
\end{array}\right) .
$$

Here, $C$ is the Dirac-Pauli charge-conjugation matrix and $\mathcal{M}$ is a $3 \times 3$ symmetric mass matrix. One may diagonalize $\mathcal{M}$ with help of a unitary matrix $U$ in the following way:

$$
U^{T} \mathcal{M U}=\operatorname{diag}\left(m_{1}, m_{2}, m_{3}\right),
$$

where $m_{1}, m_{2}$, and $m_{3}$ are real and non-negative. The physical neutrinos $\nu_{1}, \nu_{2}$, and $\nu_{3}$ are given by

$$
\left(\begin{array}{l}
\nu_{e} \\
\nu_{\mu} \\
\nu_{\tau}
\end{array}\right)=U\left(\begin{array}{l}
\nu_{1} \\
\nu_{2} \\
\nu_{3}
\end{array}\right)
$$

Then,

$$
\mathcal{L}_{\text {mass }}^{(\nu)}=\frac{1}{2} \sum_{i=1}^{3} m_{i}\left(\nu_{i}^{T} C^{-1} \nu_{i}-{\overline{\nu_{i}}}_{C}{\overline{\nu_{i}}}^{T}\right)
$$

Experiment indicates that two linearly independent squared-mass differences among the three physical neutrinos differ by a few orders of magnitude. Indeed, $\Delta m_{\text {atm }}^{2}$ is of order $10^{-3} \mathrm{eV}^{2}$, while $\Delta m_{\odot}^{2}$ may be either of order $10^{-5} \mathrm{eV}^{2}$, in the case of the MSW solution for the solar-neutrino puzzle, or of order $10^{-10} \mathrm{eV}^{2}$, in the case of the vacuum-oscillations ("just so") solution. It is customary to identify $\nu_{3}$ as the neutrino which has a mass much different from the masses of the other two, viz.,

$$
\left|m_{2}^{2}-m_{1}^{2}\right|=\Delta m_{\odot}^{2} \ll\left|m_{3}^{2}-m_{1}^{2}\right| \approx\left|m_{3}^{2}-m_{2}^{2}\right| \approx \Delta m_{\text {atm }}^{2} .
$$

Then, the negative result of CHOOZ's search for $\nu_{e}$ oscillations [1] is interpreted as $\left|U_{e 3}\right| \leq 0.217$, which is valid for $\Delta m_{\mathrm{atm}}^{2} \geq 2 \times 10^{-3} \mathrm{eV}^{2}$. 
It has been pointed out [2] that the assumption of an approximate lepton-number symmetry $\bar{L} \equiv$ $L_{e}-L_{\mu}-L_{\tau}$ (where $L_{e}$ is the electron number, $L_{\mu}$ is the muon number, and $L_{\tau}$ is the tau number) may constitute a good starting point for a model of the neutrino mass matrix. Indeed, if there are no $|\Delta \bar{L}|=2$ mass terms then

$$
\mathcal{M}=\left(\begin{array}{ccc}
0 & r b & b \\
r b & 0 & 0 \\
b & 0 & 0
\end{array}\right)
$$

where $b$ and $r$ may, without loss of generality, be taken to be real and positive. The mass matrix in Eq. (6) yields $m_{3}=0, m_{1}=m_{2}=b \sqrt{1+r^{2}}$, and

$$
U=\left(\begin{array}{ccc}
\frac{1}{\sqrt{2}} & -\frac{i}{\sqrt{2}} & 0 \\
\frac{r}{\sqrt{2\left(1+r^{2}\right)}} & \frac{i r}{\sqrt{2\left(1+r^{2}\right)}} & \frac{1}{\sqrt{1+r^{2}}} \\
\frac{1}{\sqrt{2\left(1+r^{2}\right)}} & \frac{i}{\sqrt{2\left(1+r^{2}\right)}} & -\frac{r}{\sqrt{1+r^{2}}}
\end{array}\right)
$$

This is good for the following reasons:

1. The negative result of CHOOZ's search for $\nu_{e}$ oscillations gets explained through $U_{e 3}=0$.

2. Since $4\left|U_{e 1} U_{e 2}\right|^{2}=1$, vacuum oscillations of $\nu_{e}$ with maximal amplitude would occur were $m_{1} \neq m_{2}$, opening way for the "just so" solution of the solar-neutrino problem to apply.

3. It is intuitive to expect $r$ to be close to 1 . Now, if $r=1$ then $\nu_{\mu}-\nu_{\tau}$ mixing is maximal, and this explains the atmospheric-neutrino anomaly.

On the other hand, $\bar{L}$ must be broken, because $m_{1}=m_{2}$ does not allow for oscillations between $\nu_{1}$ and $\nu_{2}$ and a solution of the solar-neutrino puzzle. A good choice, in order to avoid unpleasant majorons, would be to have $\bar{L}$ to be softly broken; this would moreover permit a natural explanation for $\Delta m_{\odot}^{2} \ll \Delta m_{\mathrm{atm}}^{2}$. This option has been suggested by Joshipura and Rindani [3]; however, in those authors' models there is no predictive power for the form of the mixing matrix $U$, a fact which impairs the immediate interest and experimental testability of those models.

In this paper I put forward a simple model with softly broken $\bar{L}$ which maintains some predictive power. The model is based on Babu's mechanism for two-loops radiative generation of the neutrino masses [4]. I remind that, in general, Babu's mechanism leads to one neutrino remaining massless; however, whereas that general mechanism cannot predict the $\nu_{e}, \nu_{\mu}$, and $\nu_{\tau}$ components of the massless neutrino, the specific model that I shall put forward retains the exact- $\bar{L}$ prediction $U_{e 3}=0$. Moreover, in my model there is a rationale for the $\nu_{e}$ oscillations of maximal amplitude, and for the tiny mass difference $\Delta m_{\odot}^{2}$, which allow a "just so" explanation of the solar-neutrino deficit; that rationale is provided by the naturalness of the assumption that the term which breaks $\bar{L}$ softly is very small.

In my model I just introduce in the scalar sector, above and beyond the usual standard-model doublet $\phi=\left(\begin{array}{cc}\varphi^{+} & \varphi^{0}\end{array}\right)^{T}$, one singly-charged singlet $f^{+}$with $\bar{L}=0$, together with two doubly-charged singlets $g^{2+}$ and $h^{2+}$, and their Hermitian conjugates. The difference between $g^{2+}$ and $h^{2+}$ lies in that the former field has $\bar{L}=0$ whereas $h^{2+}$ has $\bar{L}=-2$. The Yukawa couplings of the leptons are $\bar{L}$-invariant and are given by

$$
\begin{aligned}
& \mathcal{L}_{\mathrm{Y}}^{(1)}=-\frac{m_{e}}{v}\left(\begin{array}{ll}
\overline{\nu_{e L}} & \overline{e_{L}}
\end{array}\right)\left(\begin{array}{c}
\varphi^{+} \\
\varphi^{0}
\end{array}\right) e_{R}-\frac{m_{\mu}}{v}\left(\begin{array}{ll}
\overline{\nu_{\mu L}} & \overline{\mu_{L}}
\end{array}\right)\left(\begin{array}{c}
\varphi^{+} \\
\varphi^{0}
\end{array}\right) \mu_{R}-\frac{m_{\tau}}{v}\left(\begin{array}{cc}
\overline{\nu_{\tau L}} & \overline{\tau_{L}}
\end{array}\right)\left(\begin{array}{c}
\varphi^{+} \\
\varphi^{0}
\end{array}\right) \tau_{R} \\
& +f^{+}\left[f_{\mu}\left(\nu_{e L}^{T} C^{-1} \mu_{L}-e_{L}^{T} C^{-1} \nu_{\mu L}\right)+f_{\tau}\left(\nu_{e L}^{T} C^{-1} \tau_{L}-e_{L}^{T} C^{-1} \nu_{\tau L}\right)\right] \\
& +e_{R}^{T} C^{-1}\left[g^{2+}\left(g_{\mu} \mu_{R}+g_{\tau} \tau_{R}\right)+h^{2+} h_{e} e_{R}\right]+\text { H.c. },
\end{aligned}
$$

where $f_{\mu}, f_{\tau}, g_{\mu}, g_{\tau}$, and $h_{e}$ are complex coupling constants. Notice that, in the first line of Eq. (§), I have already taken, without loss of generality, the Yukawa couplings of $\phi$ to be flavor-diagonal; $v$ denotes the vacuum expectation value of $\varphi^{0}$. 
The scalar potential $V$ has a trivial part, $V_{\text {trivial }}$, which is a quadratic polynomial in $\phi^{\dagger} \phi, f^{-} f^{+}$, $g^{2-} g^{2+}$, and $h^{2-} h^{2+}$. Besides, $V$ includes two other terms, with complex coefficients $\lambda$ and $\epsilon$ :

$$
V=V_{\text {trivial }}+\left(\lambda f^{-} f^{-} g^{2+}+\epsilon g^{2-} h^{2+}+\text { H.c. }\right) .
$$

The term with coefficient $\epsilon$ breaks $\bar{L}$ softly. I make the following assumptions: this is the only $\bar{L}$-breaking term in the theory, and $\epsilon$ is small. These assumptions are technically natural in the sense of 't Hooft [5]. 1.

From now on I shall assume, without loss of generality, $f_{\mu}, f_{\tau}, g_{\tau}, h_{e}, \lambda$, and $\epsilon$ to be real and positive. Only $g_{\mu}$ remains, in general, complex.

The neutrino mass term $\mathcal{M}_{e \mu}$ does not break $\bar{L}$ and is generated at two-loops level by the Feynman diagram in Figure 1. A similar diagram generates $\mathcal{M}_{e \tau}$. In both cases, there is in the diagram an inner charged lepton which may be either $\mu$ or $\tau$. It is clear that the mass terms thus generated obey the relation

$$
r \equiv \frac{\mathcal{M}_{e \mu}}{\mathcal{M}_{e \tau}}=\frac{f_{\mu}}{f_{\tau}}
$$

Contrary to what happens in Zee's model [6], this ratio of mass terms is not proportional to a ratio of squared charged-lepton masses [7]. As seen before, in order to obtain maximal $\nu_{\mu}-\nu_{\tau}$ mixing one would like to have $r \approx 1$. In the present model, this means that the coupling constants $f_{\mu}$ and $f_{\tau}$ should be approximately equal. In Zee's model, on the other hand, one winds up with the rather unrealistic constraint $f_{\mu} / f_{\tau} \approx\left(m_{\tau} / m_{\mu}\right)^{2}$.

Let us check whether the diagram in Figure 1 is able to yield neutrino masses of the right order of magnitude. As we shall see later, we would like to obtain $\left|\mathcal{M}_{e \mu}\right| \approx\left|\mathcal{M}_{e \tau}\right| \approx \sqrt{\Delta m_{\text {atm }}^{2}} \sim 10^{-2}-10^{-1} \mathrm{eV}$. Now, from the diagram in Figure 1 with an inner $\tau$ one obtains

$$
\mathcal{M}_{e \mu}=-2 \lambda f_{\mu} f_{\tau} g_{\tau} m_{e} m_{\tau} \frac{I}{\left(16 \pi^{2}\right)^{2}}
$$

where

$$
\begin{aligned}
I= & \frac{1}{\pi^{4}} \int d^{4} k \frac{1}{k^{2}-m_{f}^{2}} \frac{1}{k^{2}-m_{e}^{2}} \int d^{4} q \frac{1}{q^{2}-m_{f}^{2}} \frac{1}{q^{2}-m_{\tau}^{2}} \frac{1}{(k-q)^{2}-m_{g}^{2}} \\
= & \frac{1}{2\left(m_{f}^{2}-m_{\tau}^{2}\right)} \int_{0}^{\infty} \frac{d y}{(y+1)\left(y+x_{e}\right)}\left[p \ln \frac{y+x_{g}+1+p}{y+x_{g}+1-p}-p^{\prime} \ln \frac{y+x_{g}+x_{\tau}+p^{\prime}}{y+x_{g}+x_{\tau}-p^{\prime}}\right. \\
& \left.+\left(1-x_{\tau}\right) \ln x_{g}+\left(x_{\tau}-x_{g}-y\right) \ln x_{\tau}\right] .
\end{aligned}
$$

Here, $x_{e}=m_{e}^{2} / m_{f}^{2}, x_{\tau}=m_{\tau}^{2} / m_{f}^{2}, x_{g}=m_{g}^{2} / m_{f}^{2}$, and

$$
\begin{aligned}
p & =\sqrt{\left(y+x_{g}-1\right)^{2}+4 y}, \\
p^{\prime} & =\sqrt{\left(y+x_{g}-x_{\tau}\right)^{2}+4 y x_{\tau}} .
\end{aligned}
$$

The integral in Eq. (13) is convergent and may be computed numerically.f For $m_{e}, m_{\tau} \ll m_{f}$ and $m_{g} \approx m_{f}$, one finds $I$ to be of order $m_{f}^{-2}$.

In my estimate of $\mathcal{M}_{e \mu}$ I shall therefore set $I \approx m_{f}^{-2}$. The bounds from $e-\mu-\tau$ universality in $\mu$ decay and in $\tau$ decay are $f_{\mu} / m_{f} \lesssim 10^{-4} \mathrm{GeV}^{-1}$ and $f_{\tau} / m_{f} \lesssim 10^{-4} \mathrm{GeV}^{-1}$ 叫; if one allows $f_{\mu} f_{\tau} / m_{f}^{2}$ to be as high as $10^{-8} \mathrm{GeV}^{-2}$, then one obtains

$$
\left|\mathcal{M}_{e \mu}\right| \approx 10^{-15} \lambda g_{\tau}
$$

It is reasonable to assume that the Yukawa coupling $g_{\tau}$ is of the same order of magnitude as the Yukawa couplings $f_{\mu}$ and $f_{\tau}$, and that the dimensionful scalar-potential coupling constant $\lambda$ is of the same order

\footnotetext{
${ }^{1}$ Notice that the possible $\bar{L}$-breaking term $f^{-} f^{-} h^{2+}$ has dimension higher than the one of $g^{2-} h^{2+}$, and therefore the assumption of its absence is natural.

${ }^{2}$ It is not possible to use the approximations $m_{e}=m_{\tau}=0$ becquse they lead to infrared divergences. This is not a problem, since those divergences are logarithmic and $\mathcal{M}_{e \mu}$ in Eq. (11) also includes a factor $m_{e} m_{\tau}$.
} 
of magnitude as both $m_{f}$ and $m_{g}$. This leads to $g_{\tau} / \lambda \sim f_{\mu} / m_{f} \sim 10^{-4} \mathrm{GeV}^{-1}$. Fortunately the product $\lambda g_{\tau}$ stays free. In order to obtain $\left|\mathcal{M}_{e \mu}\right| \sim 10^{-2} \mathrm{eV}$ it is then sufficient to assume

$$
\begin{aligned}
\lambda \approx m_{g} \approx m_{f} & \sim 10^{4} \mathrm{GeV}, \\
f_{\mu} \approx f_{\tau} \approx g_{\tau} & \sim 1 .
\end{aligned}
$$

Extra factors of order 1 may easily enhance $\left|\mathcal{M}_{e \mu}\right|$ and bring it up to the desired value $0.06 \mathrm{eV}$.

The assumption, made in Eq. (18), that the Yukawa couplings are of order 1, may seem unrealistic. ${ }^{3}$ However, there are no experimental indications against this possibility when the masses of $f^{+}$and of $g^{2+}$ are assumed to be as high as $10 \mathrm{TeV}$. I For instance, $g^{2+}$ mediates the unobserved decay $\tau^{-} \rightarrow \mu^{-} e^{+} e^{-}$; however, by comparing that decay with the standard $\tau^{-} \rightarrow \mu^{-} \bar{\nu}_{\mu} \nu_{\tau}$, one easily reaches the conclusion that $\mathrm{BR}\left(\tau^{-} \rightarrow \mu^{-} e^{+} e^{-}\right)$should be at least one order of magnitude below the present experimental bound, when $m_{g} \approx 10 \mathrm{TeV}$ and $\left|g_{\mu} g_{\tau}\right| \approx 1$. A more complicated process is $e^{+} e^{-} \rightarrow \tau^{+} \tau^{-}$, which is mediated by $g^{2+}$ exchange in the $t$ channel. The amplitude $A$ for this process is

$$
\begin{aligned}
A= & \frac{i e^{2}}{s}\left[\bar{v}(e) \gamma^{\mu} u(e)\right]\left[\bar{u}(\tau) \gamma_{\mu} v(\tau)\right]+\frac{i e^{2}}{3\left(s-m_{z}^{2}\right)}\left[\bar{v}(e) \gamma^{\mu} \gamma_{5} u(e)\right]\left[\bar{u}(\tau) \gamma_{\mu} \gamma_{5} v(\tau)\right] \\
& -\frac{i g_{\tau}^{2}}{8\left(t-m_{g}^{2}\right)}\left[\bar{v}(e) \gamma^{\mu}\left(1+\gamma_{5}\right) u(e)\right]\left[\bar{u}(\tau) \gamma_{\mu}\left(1+\gamma_{5}\right) v(\tau)\right]
\end{aligned}
$$

I have used the convenient approximations $m_{e}=m_{\tau}=0$ and $\sin ^{2} \theta_{w}=1 / 4$ in writing down the standardmodel amplitude, and a Fierz transformation in the non-standard contribution. If one defines $j=2 \mathrm{~m}_{g}^{2} / \mathrm{s}$, $z=g_{\tau}^{2} /\left(2 e^{2}\right)$, and $l=3\left(s-m_{z}^{2}\right) / s$, then one finds

$$
\frac{d \sigma}{d \cos \theta} \propto \frac{l^{2}+1}{l^{2}}\left(1+\cos ^{2} \theta\right)+\frac{4}{l} \cos \theta+z \frac{l+1}{l} \frac{(1+\cos \theta)^{2}}{1+j+\cos \theta}+z^{2} \frac{(1+\cos \theta)^{2}}{(1+j+\cos \theta)^{2}},
$$

where $\theta$ is the angle between the momenta of $e^{-}$and of $\tau^{-}$in the center-of-momentum frame. From the differential cross section in Eq. (20) one easily checks that the deviations of both the total cross section and the forward-backward asymmetry from their standard-model predictions are completely negligible when $m_{g} \sim 10 \mathrm{TeV}$, even if $g_{\tau}$ is as large as 1 .

Except for $\mathcal{M}_{e \mu}$ and $\mathcal{M}_{e \tau}$, all other matrix elements of $\mathcal{M}$ break $\bar{L}$ and, therefore, they will all be proportional to the $\bar{L}$-breaking parameter $\epsilon$, which is assumed to be small. The matrix elements $\mathcal{M}_{\mu \mu}$, $\mathcal{M}_{\mu \tau}$, and $\mathcal{M}_{\tau \tau}$ arise at two loops from the diagram in Figure 2. In order to obtain a non-zero $\mathcal{M}_{e e}$ one must go to three loops and use for instance the diagram in Figure 3. In that diagram there are two inner charged leptons which may be either $\mu$ or $\tau$; therefore, there is a contribution to $\mathcal{M}_{e e}$ proportional to $m_{\tau}^{2}$, and that matrix element should not be neglected in spite of it only arising at three-loops level.

The diagram in Figure 2 clearly leeds to the following relation:

$$
\mathcal{M}_{\mu \mu}: \mathcal{M}_{\mu \tau}: \mathcal{M}_{\tau \tau}=f_{\mu}^{2}:\left(f_{\mu} f_{\tau}\right): f_{\tau}^{2}=r^{2}: r: 1 .
$$

One thus obtains that in the present model

$$
\mathcal{M}=\left(\begin{array}{ccc}
a & r b & b \\
r b & r^{2} c & r c \\
b & r c & c
\end{array}\right),
$$

where $a, b$, and $c$ are complex numbers with mass dimension, while $r=f_{\mu} / f_{\tau}$ is a real dimensionsless number which should in principle be of order 1 . The masses $a$ and $c$ are suppressed relative to $b$ by the soft-breaking parameter $\epsilon$.

The mass matrix in Eq. (22) immediately leads to two predictions of this model: there is one massless neutrino $\left(\nu_{3}\right)$ and its component along the $\nu_{e}$ direction, i.e., $U_{e 3}$, vanishes. Indeed, the diagonalizing

\footnotetext{
${ }^{3}$ Notice however that, in the standard model, the top-quark Yukawa coupling is also very close to 1.

${ }^{4}$ Concerns about the breakdown of perturbativity are only justified for Yukawa couplings $\gtrsim 4 \pi$, i.e., of order 10 or more.
} 
matrix $U$ reads

$$
U=\left(\begin{array}{ccc}
\cos \psi & -i \sin \psi & 0 \\
e^{i \alpha} \frac{r \sin \psi}{\sqrt{1+r^{2}}} & e^{i \alpha} \frac{i r \cos \psi}{\sqrt{1+r^{2}}} & \frac{1}{\sqrt{1+r^{2}}} \\
e^{i \alpha} \frac{\sin \psi}{\sqrt{1+r^{2}}} & e^{i \alpha} \frac{i \cos \psi}{\sqrt{1+r^{2}}} & -\frac{r}{\sqrt{1+r^{2}}}
\end{array}\right) \cdot \operatorname{diag}\left(e^{i \theta_{1}}, e^{i \theta_{2}}, 1\right)
$$

cf. Eq. (7). In the matrix of Eq. (23) $\alpha \equiv \arg \left[a b^{*}+b c^{*}\left(1+r^{2}\right)\right]$ is a physically meaningless phase. The Majorana phases $\theta_{1}$ and $\theta_{2}$ are necessary in order to obtain real and positive $m_{1}$ and $m_{2}$. The sole physically observable phase is $2\left(\theta_{1}-\theta_{2}\right)[8$. The mixing angle $\psi$ is given by

$$
\tan \psi=\sqrt{1+\varepsilon^{2}}+\varepsilon
$$

where

$$
\varepsilon=\frac{|c|^{2}\left(1+r^{2}\right)-|a|^{2}}{2 \sqrt{1+r^{2}}\left|a b^{*}+b c^{*}\left(1+r^{2}\right)\right|}
$$

is a parameter of order $\epsilon$, just as $a / b$ and $c / b$, and may therefore be assumed to be very small. Thus, $\psi$ is close to $45^{\circ}$. The amplitude of the vacuum oscillations of $\nu_{e}$ relevant for the solution of the solar-neutrino problem is $4\left|U_{e 1} U_{e 2}\right|^{2}=\left(1+\varepsilon^{2}\right)^{-1}$, i.e., almost maximal. Thus, the present model favors a "just so" solution of the solar-neutrino puzzle.

The soft-breaking parameter $\epsilon$ should be tiny. Indeed, one finds

$$
\frac{\Delta m_{\odot}^{2}}{\Delta m_{\mathrm{atm}}^{2}} \approx 2 \frac{\left|a b^{*}+b c^{*}\left(1+r^{2}\right)\right|}{|b|^{2} \sqrt{1+r^{2}}} \sim \epsilon
$$

as we want the "just so" solution for the solar-neutrino puzzle to apply, we must accept $\epsilon$ to be of order $10^{-7}$. Such a tiny soft breaking of $\bar{L}$ may eventually be explained by some new physics at a very high energy scale.

From the non-observation of neutrinoless double beta decay one derives the bound $\left|\mathcal{M}_{e e}\right| \leq 0.2 \mathrm{eV}$ [9]. This is not a problem to the present model. Indeed, as $m_{3}$ is predicted to vanish, $m_{1}$ and $m_{2}$ should both be very close to $\sqrt{\Delta m_{\mathrm{atm}}^{2}} \approx 0.06 \mathrm{eV}$. Thus, in the approximation $\cos ^{2} \psi=\sin ^{2} \psi=1 / 2$, one has

$$
\left|\mathcal{M}_{e e}\right| \approx(0.03 \mathrm{eV})\left|e^{2 i\left(\theta_{1}-\theta_{2}\right)}-1\right|<0.2 \mathrm{eV} .
$$

Moreover, the phase $2\left(\theta_{1}-\theta_{2}\right)$ is very close to zero - indeed, it vanishes in the limit of $\bar{L}$ conservation.

In conclusion, the model that I have presented in this paper makes the exact predictions $m_{3}=0$ and $U_{e 3}=0$, while it naturally accomodates maximal amplitude $\nu_{e}$ oscillations and a tiny $\Delta m_{\odot}^{2}$. Maximal $\nu_{\mu^{-}}$ $\nu_{\tau}$ mixing follows from the reasonable assumption that two Yukawa couplings are almost equal. Neutrino masses are small because they are radiatively generated at two-loops level. Indeed, the fact that two neutrino masses are as large as $0.06 \mathrm{eV}$ practically forces the new mass scale, at which the extra scalars lie, to be in the $10 \mathrm{TeV}$ range; while deviations from $e-\mu-\tau$ universality in $\mu$ decay and in $\tau$ decay should be close at hand. The model requires some physical mechanism for generating a tiny soft breaking of $\bar{L}$.

It is a pleasure to thank Walter Grimus for his generous help and valuable advice, and for reading and criticizing two drafts of the manuscript. I also thank Evgeny Akhmedov for calling my attention to a potential problem with the model, and for reading the final version.

\section{References}

[1] CHOOZ Collaboration (M. Apollonio et al.), Phys. Lett. B 466, 415 (1999).

[2] R. Barbieri, L. J. Hall, D. Smith, A. Strumia, and N. Weiner, JHEP 9812, 017 (1998). 
[3] A. S. Joshipura, Phys. Rev. D 60, 053002 (1999); A. S. Joshipura and S. D. Rindani, hep-ph/9811252 (Eur. Phys. J. C, to be published).

[4] K. S. Babu, Phys. Lett. B 203, 132 (1988).

[5] G. 't Hooft, in Recent developments in gauge theories, Cargèse 1979 (ed. G. 't Hooft et al.). Plenum Press, New York, 1980.

[6] A. Zee, Phys. Lett. 93B, 389 (1980).

[7] C. Jarlskog, M. Matsuda, S. Skadhauge, and M. Tanimoto, Phys. Lett. B 449, 240 (1999).

[8] See for instance G. C. Branco, L. Lavoura, and J. P. Silva, CP violation (Oxford University Press, New York, 1999), page 305.

[9] L. Baudis et al., Phys. Rev. Lett. 83, 41 (1999).

\section{Figure captions}

Figure 1: Two-loops Feynman diagram which generates $\mathcal{M}_{e \mu}$.

Figure 2: Two-loops Feynman diagram which generates $\mathcal{M}_{\mu \tau}$.

Figure 3: One of the three-loops Feynman diagrams which generate $\mathcal{M}_{e e}$. 


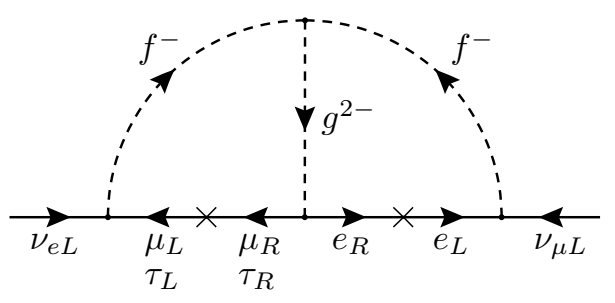

Figure 1

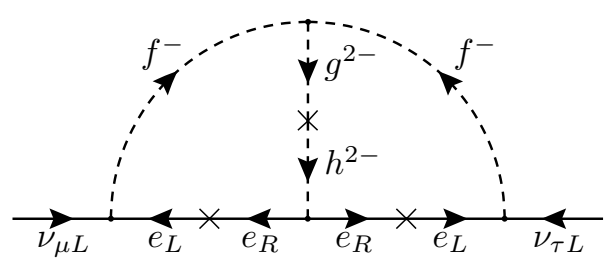

Figure 2

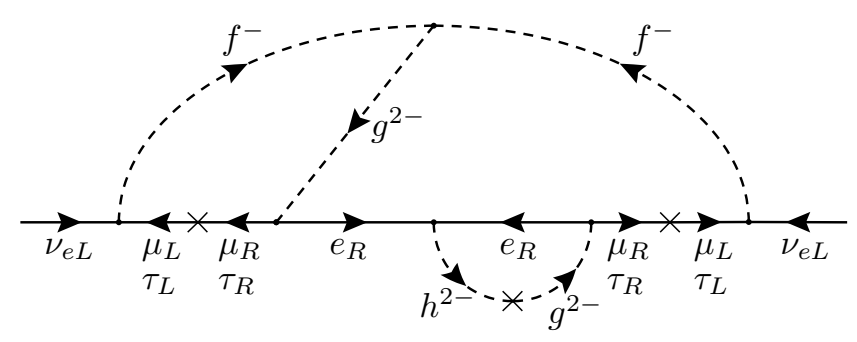

Figure 3 